Southern Illinois University Carbondale

OpenSIUC

Articles

Information \& Communication Sciences

2-24-2021

\title{
Adapting to Remote Library Services during COVID-19.
}

Lydia A. Howes

Lynne Ferrell

Geoffrey S. Pettys

Adam Roloff

Follow this and additional works at: https://opensiuc.lib.siu.edu/ics_articles

"This is an Accepted Manuscript of an article published by Taylor \& Francis in Medical

Reference Services Quarterly in February 2021, available online:

http://www.tandfonline.com/10.1080/02763869.2021.1873616

This Article is brought to you for free and open access by the Information \& Communication Sciences at OpenSIUC. It has been accepted for inclusion in Articles by an authorized administrator of OpenSIUC. For more information, please contact opensiuc@lib.siu.edu. 


\title{
Adapting to Remote Library Services During COVID-19
}

\author{
Lydia Howes* \\ Lynne Ferrell \\ Geoff Pettys \\ Adam Roloff
}

\begin{abstract}
In response to the COVID-19 pandemic and the subsequent Stay At Home order, the Southern Illinois University Medical Library utilized new technologies and implemented new virtual service models in order to improve internal communication, and to continue providing services and resources to patrons remotely. The changes happened quickly and the librarians faced several challenges during this time, but things went smoothly overall and there were some considerable silver linings. Several of the newly adopted technologies, service models, and virtual resource offerings proved to be extremely effective and their use will continue beyond the duration of the pandemic.
\end{abstract}

KEYWORDS: COVID-19; Reference Services; Interlibrary Loan; Virtual Services

\author{
AUTHORS \\ Lydia Howes, MSI (lhowes44@ siumed.edu) is an Assistant Research and Education Librarian at \\ the Southern Illinois University Medical Library, 801 N Rutledge St., Springfield, IL 62794- \\ 9625, USA.
}


Lynne Ferrell, MSLIS (lferrel191@ siumed.edu) is Head, Scholarly \& Institutional Resources at Southern Illinois University Medical Library, 801 N Rutledge St., Springfield, IL 62794-9625, USA.

Geoff Pettys, MA, MSLIS, AHIP (gpettys31@ siumed.edu) is Head of Reference \& Education Services at Southern Illinois University Medical Library, 801 N Rutledge St., Springfield, IL 62794-9625, USA.

Adam Roloff, MLIS (aroloff47@ @iumed.edu) is an Assistant Research and Education Librarian at the Southern Illinois University Medical Library, 801 N Rutledge St., Springfield, IL 627949625, USA.

\section{ORCID}

Lydia Howes- 0000-0002-7769-2717

Lynne Ferrell- 0000-0002-4061-6294

Geoff Pettys- 0000-0003-1826-383X

Adam Roloff- 0000-0003-3295-0687

\section{INTRODUCTION}

The Southern Illinois University School of Medicine (SIU-SOM) is a community-based medical school located in Springfield, Illinois. It was established in 1970 to educate physicians and to encourage graduates to remain in central and southern Illinois. SIU-SOM is part of the Southern Illinois University System (SIU) and is overseen by the Chancellor located in Carbondale, IL. The Dean of the School of Medicine also serves as the School's Provost and oversees the various 
departments within the school. The Medical School includes medical, residency, and fellowship programs; a Medical/Dental Educational Preparatory Program; a Physician Assistant Program; two graduate degrees in Medical Microbiology/Immunology/Cell Biology and Neuroscience/Pharmacology; and a clinical branch called SIU Medicine. The Library currently serves around 550 students, 300 residents and fellows, and 1700 full-time faculty and staff.

The Physician Assistant Program and the first year of Medical School are based in the Carbondale campus. First year medical students make use of SIU Carbondale's facilities for the basic science curriculum then move to the Springfield campus for their final three years where, with two community hospital partners, clinical education can be best supported. The school has a fully functional Medical Library on the main campus in Springfield and a smaller Medical Resource Center to serve the students, faculty, and staff in Carbondale.

The Medical Library has 18 staff members, including five library faculty and the nonfaculty Director, to assist students, residents, fellows, faculty, and staff in their educational and research needs. The Medical Library consists of three departments: Reference, Circulation, and Technical Services. The Reference team includes four faculty: the Reference Supervisor, Outreach Librarian, Education Librarian, and Technology Coordinator. The fifth library faculty member, the Scholarly \& Institutional Resources Librarian, heads the Library's Technical Services department.

\section{TIMELINE}

As the COVID-19 pandemic gained attention in the United States, SIU took action by implementing a system-wide restriction on travel on March 6, 2020. The Dean and Provost of SIU-SOM and SIU Medicine, the clinical practice of the school, also immediately began 
planning and implementing measures to mitigate staff exposure. By March 11, SIU Medicine had implemented new protocols and safety procedures for clinicians, staff, and patients; the Medical Library followed suit and limited access to the common computer area to ensure social distancing. The Medical Library also took the additional step of restricting public access to the library.

On March 13, SIU distributed new work-from-home policies and procedures to staff and faculty, and announced that all classes system-wide would go virtual following Spring Break. For many years the School has encouraged faculty to move lectures to online or flippedclassroom formats and the Medical Library has assisted in these efforts. The Library CAVE (Create Audio and Video for Education) lab contains the only publicly available computers on campus equipped with hardware and software dedicated to creating instructional video or online modules. As such, the Medical Library received a large number of requests from SIU-SOM faculty to help create video lectures to meet the new virtual class requirements.

On March 17, most library employees started working remotely. Only a few library faculty and staff remained on-site to help faculty finish their video lectures. All library employees were working remotely by the end of the day on March 20, when the Illinois Governor issued a Stay At Home order, effective March 21. While the clinical branch and SIUSOM's campus buildings largely remained open with precautions, most School and non-clinical employees worked from home. Medical students and residents retained 24-hour access to the study and common computer areas in the Library. The physical collection, which has always been unavailable to patrons outside of staffed hours, remained inaccessible throughout the workfrom-home period. Library employees maintained a remote work schedule until June 1, when the library reopened to SIU-SOM affiliates with reduced hours and transitioned some staff back on- 
site. Currently, staff work-from-home or on-site in two week rotations; this will extend indefinitely.

\section{GOING VIRTUAL}

\section{Reference Services}

The Reference team continued service while working remotely, and was ultimately successful in finding virtual alternatives for almost all regularly offered services. Working from home, the team assisted patrons in finding full-text articles and learning to use electronic resources, provided scientific editing services, conducted virtual workshops on using EndNote, and completed literature searches. As it was particularly challenging to provide virtual training and troubleshoot patrons' technology problems, SIU-SOM's IT department authorized librarian use of ConnectWise (a program that provides the ability to both view and control a patron's computer remotely). Library faculty used Webex and Zoom videoconferencing software to

provide instructional workshops and one-on-one consultations. During the 10 weeks prior to the shut-down, the Medical Library received a total of 163 reference requests, out of which $42 \%$ were received by email, $42 \%$ in person, and $16 \%$ by phone. During the 10 -week work-fromhome period, all SIU-SOM employees were instructed not to forward office phones to personal numbers, so phone requests dropped along with in-person requests. While the Library was operating remotely, $96 \%$ of the 105 requests were handled via email, $3 \%$ by "in-person" video chats, and $1 \%$ by phone. Since reopening the Library, in-person requests have increased, but, unsurprisingly, have not returned to pre-COVID levels. From August 23 to October 31, 56\% of 95 requests were made via email, $23 \%$ in-person, and $21 \%$ by phone. 
Significantly, during the shut-down the total number of reference requests fell to around $44 \%$ of the requests received during the same period in 2019 , and have not greatly increased since the Library reopened. A small portion of the drop may be attributed to team members failing to track reference interactions during this period of upheaval, but the COVID-19 pandemic is likely the major player in this decrease; prior to the shut-down, the number of reference requests had been trending higher compared to the previous year. The Library's literature search service has gained popularity over the past several years, and demand continued to increase during the pandemic. The workflow for literature searches during the work-from-home period remained similar to when working on-site. Requests are received by reference staff on duty via email through a webform and assigned to a librarian, who completes the search and emails it back to the patron. During the Stay At Home period, the school-wide availability of Microsoft's Remote Desktop Connection allowed library faculty to see and access databases exactly as they would on campus. In lieu of in-person meetings, Webex conferences were offered to researchers to review literature search results and discuss any questions they had.

The Reference team had anticipated a surge in requests for literature searches following the Governor's Stay At Home order, as so many faculty and staff were suddenly working remotely with newly found time to do research. Within weeks of the Governor's order, it was clear the hypothesis was correct. While there is typically a slight decrease in literature search requests in May and June, in May 2020 (the second full month of the Stay At Home order), the Library set a new record for the greatest number of literature search requests received in a single month with 10 unique requests. This was a 100\% increase compared to the previous month, and a striking 233\% increase compared to May 2019 (See Figure 1). While the service has steadily gained popularity in fiscal year 2020, making it difficult to assess whether COVID impacted 
these numbers, the large increase in May was unprecedented. Further, the Reference team usually receives a spike in requests starting in late summer and continuing into the fall; in 2020 that spike seems to have occurred a few months earlier than usual, with another dramatic increase occurring in October. While it is difficult to prove causation, the data show that the Reference team received nearly as many literature search requests during the first four months of working remotely as it did in all of the previous fiscal year (July 2018 - June 2019).

\section{[PLACE FIGURE 1 HERE]}

\section{LEGEND FIGURE 1: LITERATURE SEARCH REQUESTS RECEIVED, BY MONTH}

\section{Interlibrary Loan}

The SIU Medical Library maintains membership in a variety of consortia and associations, and also provides document delivery services to SIU Medicine staff, Medline Industries, CVS/Caremark, international libraries, and other libraries or businesses in Illinois and Springfield as requested. As COVID spread, many libraries in Illinois and throughout the country simply closed and suspended their interlibrary loan functions. The SIU Medical Library was one of few libraries that continued to process ILL requests.

While working remotely, the Interlibrary Loan department experienced an increase in lending requests. Many campus faculty used this time to request articles to complete ongoing research projects and papers. As more libraries discovered that the SIU Medical Library remained open, requests accelerated via both DOCLINE and OCLC, and international requests tripled. In June, the Library received 16 international requests, which is more than had been 
received in the past three years. On the downside, unfilled lending requests in April and May reached a total high of over 650 out of approximately 1000 total requests, as library staff working remotely could not physically access the Library's print collections, including books or bound volumes of serial titles.

\section{GoSkills Online Tutorials}

GoSkills, a subscription-based service that provides access to online training modules on a variety of topics and common software, proved to be extremely popular during the shut-down. The Library has subscribed to GoSkills since July 2019, when it replaced the previous e-learning subscription. The Library had a 20 user-license package from GoSkills. During the work-fromhome period the Dean and Provost of the School advertised the service in his weekly emails, which increased interest. The Library saw an overall increase of 10\% from March-August compared to the previous six months of September-February, and had to purchase additional licenses to meet demand. It proved to be a very useful learning opportunity for many SIU-SOM employees with regular duties that were not conducive to remote work, including some library staff.

\section{COVID-19 LibGuide}

Shortly before all library staff started working remotely, the Outreach Librarian created a Coronavirus/COVID-19 LibGuide ${ }^{1}$. Maintaining the LibGuide proved to be a challenge, as the information changed frequently and many resources required evaluation prior to inclusion. The intent of the LibGuide was largely to provide information to non-affiliated SIU-SOM users and the general public, due to the Library being an Outreach Partner Library with the Network of the 
National Library of Medicine. As such, specific internal SIU-SOM protocols and guidelines were excluded from the LibGuide. That information was being highly marketed to staff, students, and faculty through the school's intranet postings and daily email updates. Priority for inclusion on the LibGuide was given to the Center for Disease Control, Johns Hopkins University, the National Library of Medicine, and the Illinois Department of Public Health. Other resources added to the guide were quickly evaluated based on a set of criteria: value of the information, originality, and accessibility. Not all the information for every resource could be vetted, but the criteria served as a means to quickly evaluate a resource.

The LibGuide was divided into four sections: Coronavirus Resources (general resources), Clinician Resources (specifically for clinicians and healthcare staff), Infographics and Videos (containing quick facts and information videos), and a section devoted to the CDC COVID-19 Microsite. From March 14 to when the Library reopened June 1, the COVID-19 LibGuide was the third most viewed guide, with 149 views, following guides on Posters and Presentations (275) and Creating Educational Videos (176), and followed by [Library] Class Descriptions and Handouts (117) and Consumer Health Information (88). It was the most viewed guide for two days shortly after being released, on March 16 and 17.

\section{Orientation}

In the past, the Library has offered a lunch hour orientation session and tour during the Year 2 (Y2) Medical Student orientation in early August. Given restrictions on in-person events, this year the Library chose to create two quick video tours: a video walkthrough of the Medical Library's physical space, and a website overview recorded using TechSmith's Camtasia. Links to these videos, along with some fact-sheets and information concerning creating library accounts, 
were distributed to the incoming Y2 class via email. Based on views, approximately half of the Y2 students watched the orientation videos (See Table 1).

\section{WORKING FROM HOME}

\section{Microsoft Teams}

Working from home resulted in a remarkable increase in the quantity of email being sent and received. Questions that normally would have been asked quickly in person suddenly required multiple emails, often with more recipients than would normally be the case. Keeping up with the constant deluge of emails was time consuming and seemed inefficient. Within the first few days of working from home, the Education Librarian (EL) suggested the department instead try using Microsoft Teams, which is provided by SIU, as a means of remaining in communication. The Reference team adapted to Teams almost immediately and the response was overwhelmingly positive. Not only was Teams successful in minimizing the number of emails sent, but using this chat platform had several additional and unanticipated results.

Teams provided a virtual alternative to the impromptu discussions and brainstorming sessions that were common during the course of a typical workday. The Teams chat served as a virtual replacement for "office pop-ins" and unscheduled discussions. In some ways, the virtual

alternative proved to be better than in-person because faculty could post and respond to questions at their convenience. The fear of interrupting someone's workflow was minimized, as faculty were able to finish what they were doing and then respond to the chat.

The Reference department also used Teams to proofread responses to patron emails and offer editing suggestions. With communication being done almost exclusively via email, and knowing that tone and intention is often misinterpreted without the benefit of vocal inflection 
and non-verbal cues, the Reference team would frequently paste email drafts in the Teams chat for others to review. In almost every instance of this "peer-reviewed messaging" process, suggestions were made that resulted in better and clearer email communication.

One of the most positive and unexpected outcomes of using Teams while working remotely was that it seemingly brought the Reference staff closer together as a unit. The team actually communicated more than they did while working on-site. Teams provided an informal, yet effective platform to brainstorm, work through problems, ask for and provide feedback, and simply check in on each other. While it seems a bit unnecessary for a centrally-located, fourperson division, the Reference staff unanimously decided to continue using Teams when staffing and workflows return to normal.

\section{ADDRESSING CHANGING NEEDS}

\section{Online Instruction through Video Tutorials}

Prior to the pandemic, the Reference team had discussed developing interactive instructional videos on library-related topics, but no one had ever found time to actually do so. The shut-down reinforced the importance of having such resources, and the change to work-from-home provided the opportunity for the long blocks of uninterrupted time required to develop, record, and edit a tutorial series.

The first several videos developed during this time were mostly an afterthought. While assisting faculty frantically working to record video lectures, the EL recognized that there was much larger demand than could be met with the Library's limited resources. They recorded a short video demonstrating how to record PowerPoint lectures, using only PowerPoint and a microphone. The EL and the Technology Coordinator (TC) then worked together to update the 
existing "Creating Educational Videos" LibGuide ${ }^{2}$ and printed instructional handouts. The Library Director distributed these resources to SIU-SOM faculty on March 18 and several faculty with CAVE appointments instead opted to record lectures using these instructions. The tutorial is still consistently recommended and viewed. While working from home the TC and EL also quickly pulled together a PubMed course in MyCourses, SIU-SOM's Moodle course management system, by loading the NNLM's PubMed Essentials tutorial series ${ }^{3}$, supplemented with two short videos recorded with TechSmith's SnagIt instructing users how to 1) access PubMed from the Library site, and 2) use the "Find It @ SIU-SOM" button to access paid library resources from off-campus. This beta test was not widely distributed, but it did demonstrate that recording videos from home would be possible and it might finally be the time to develop training modules! The Library already had several licenses to TechSmith's Camtasia, so SIUSOM's IT transferred one to an institutional laptop used by the EL.

\section{Finding Full Text}

The first video series, "Finding Full Text" was created entirely during the Stay At Home order, and demonstrated using the Library website to access journal articles. Prior to the shut-down, requests for articles easily accessible through the Library's online collections and questions concerning off-campus access to electronic resources made up a significant portion of reference questions received. When a large portion of the School's staff made the switch to work-fromhome, it became obvious that the Reference team had underestimated the number of patrons who did not know how to access resources from off-campus.

It took about a week to script, storyboard, and record a series of 4 videos. Initial recordings were completed in in the EL's bathroom (the only room without street noise) using 
Camtasia and the laptop's built in microphone. Following the initial recording phase, the EL spent roughly four working days editing the videos (approximately one day for each video). As each was completed, the EL sent these first drafts to the rest of the team to view and make suggestions. After several rounds of revisions, the Reference team asked the Circulation team for their feedback.

When the modules were completed they were uploaded to screencast.com, TechSmith's cloud storage solution, and embedded in an existing "Finding Full Text" LibGuide ${ }^{4}$. From start to finish it took roughly one month to script, record, and edit the entire series: Using the Citation Linker, Using the e-Journals A-Z List, Using Databases and the Find It @ SIU-SOM Link, and Using SIU Carbondale Resources.

The original intent was to keep the videos to around five minutes each; however, the last two videos run longer. In an effort to increase engagement and retention, each includes several interactive features that require the viewer to click or select the appropriate links in order to continue the tutorial. Each video ends with an optional three question quiz.

\section{EndNote}

The Library's EndNote class has been very popular, to the point where it was the only workshop taught remotely (via Webex) during the shut-down. During the school year, the class is offered monthly, yet patrons often request special sessions or one-on-one consultations. Students and residents have a tendency toward waiting to install and learn to use EndNote the week before a deadline and don't want to wait for the next scheduled workshop. Reference staff often directed patrons asking for training materials to Clarivate's YouTube channel and SIU Carbondale's 
fairly robust LibGuide; unfortunately, these rarely seemed to provide the foundation patrons needed. The reference team decided a video tutorial series might be a good solution.

Development for this series began around the time the library reopened on June 1 with rotating, 50\% staffing, so the EL recorded this in the CAVE and edited it partially in the CAVE and partially while working from home. Again, the Reference and Circulation teams each provided one round of feedback, and the finished videos were uploaded to screencast.com and embedded on the Medical Library's EndNote LibGuide'.

Like the Finding Full Text series, each video includes some interactivity and quiz questions. The videos in the EndNote series are longer, so they also include a clickable Table of Contents to allow viewers to jump to a specific section. This series is five videos long: Getting Started with EndNote, Importing References, Organization and Resource Management, EndNote Online- Syncing \& Sharing Libraries, and Creating Citations \& Bibliographies.

\section{[PLACE TABLE 1 HERE]}

\section{LEGEND TABLE 1: VIDEOS DEVELOPED DURING COVID-19}

Now that the videos have been created, the Reference team continues to contemplate how to make them easier to find. The main SIU-SOM site only allows embedded video from YouTube, which would not support interactive elements. LibGuides are the only section of the Medical Library website that can host the tutorials as designed. Unfortunately, due to the structure of the SIU-SOM website, the Library's LibGuides are not easily discoverable. To increase visibility, the tutorial series were announced via banners on the Library's website, added to the website's Key Resources page, and featured in the Library's August newsletter. While 
individual views of videos were not accurately tracked until July 31 (when the Library linked Google Analytics to screencast.com), LibGuide views indicate that these promotions did increase traffic to the relevant guides.

\section{Expanding Electronic Resources}

With a goal to decrease print serial subscriptions overall, a large percentage of the Library's serials were renewed in online format for 2020 . This reduced print serials to less than $5 \%$ of the collection, so users continued to have current online serial resources available. However, outside of e-books available in Elsevier's Clinical Key, the Library had very few e-books. The majority of books purchased since 2019 were print books, and most of those purchases were for new editions of Reserve books. Reserve books include preparatory exam books, the most highly referenced and utilized text books, and basic science, pathology and diagnostic books used by medical students. When the Library closed, students and faculty placed numerous requests for books the Library only had in print and that could not circulate during shut-down. It became obvious that the Library did not have an adequate collection of e-books to cover Reserve topics or supplement virtual classrooms.

As publishers and vendors became aware of the far-reaching effects library closures had on access to resources, they bombarded the Scholarly \& Institutional Resources Librarian (SIRL) with emails. They made collections of COVID research freely available, and offered temporary access, trials, or other special offers for virtual learning resources and materials. This was an opportune time for trialing other products or packages with little risk and no financial expense. Many of the offers appeared generic, and were not appropriate for an academic health science research library to explore further. 
On March 25, the SIRL received an email which offered a complimentary trial to Access Medicine. On April 9, the Medical Library was granted 30 day trial access to not only Access Medicine, but also a bevy of their other platforms: Access Pharmacy, Access Anesthesiology, Access Cardiology, Case Files Collection, Teaching Case Files, Access Pediatrics, Access Surgery, JAMA Evidence, Access Emergency Medicine, and later, as a direct request from one of the Oncology physicians, Access HemOnc. Each Access platform was added to the Library's web page, noting that complementary access was available until May 1.

Because the SIU Physician Assistant Program is in Carbondale and medical students spend their first year located in Carbondale, the Medical Library was able to disseminate trial information to the Medical Resource Center Director. Thus, students could access platforms via the Library website. Feedback was extremely favorable; the Library received emails from students, PAs and physicians stating how excellent, useful and "life-saving" these online texts were. They also requested extending trials if possible. In mid-April, the Library received confirmation to extend the trials through June 1. It was evident from usage statistics that specific resources were being heavily relied on, therefore only the 6 most used resource trials were extended.

In early June, when the Library Director requested a quote for an Access Medicine subscription, McGraw Hill conveyed that they would provide another platform free for the first year if Access Medicine was purchased by July 1. It was quickly decided the Library would subscribe to Access Medicine and Case Files, which was by far the highest use resource after Access Medicine. McGraw Hill also offered to extend their trials through July 4 while negotiating the agreement. This collaboration with McGraw Hill was extraordinary. 
On the flip side, a prominent vendor offered trials to several of their products that SIUSOM students could benefit from; however, the Library was never able to configure access to those products. The Library and SIU-SOM IT worked with the vendor's support for months, but the issue was never resolved. It was very unfortunate, challenging and still unclear exactly why the Library could not connect to the vendor's freely available "trial" databases.

\section{WHAT'S NEXT}

At the time of writing, the SIU-SOM library staff is working in two-week rotations, with half the staff working on-site and the other half working from home. The library will remain closed to the public for the foreseeable future, but is open with reduced hours for SIU affiliates. Reference staff working on-site provide in-person services, albeit with some modifications to ensure social distancing. The shared reference and circulation desk is protected by wheeled plexiglass panels, and when possible patrons are instructed to ask questions or place requests via email or phone. Conference rooms, the Library's computer training lab, and the CAVE are again reservable, but with limited side-by-side assistance or instruction and reduced capacity. Capacity limits are set by SIU-SOM administration and change based on the current mitigation protocols. Masks are required and provided along with hand sanitizer and disinfecting wipes. On-site staff take on the majority of reference duties, including responding to emails, phone calls, and any drop-in reference requests. While off-site, they focus on project based work, as the Reference team feels strongly that working from home is more conducive to greater productivity for projects. While the "expected interruptions" from reference duties are a vital and enjoyable part of a reference librarian's work, they can make it difficult to focus on projects due to the disruption in work flow and concentration. 
Moving forward, the Reference team will continue to create virtual alternatives to services that would otherwise be provided in person. The video tutorials and tours that were created during the Stay At Home order have proven to be extremely popular. Whereas the monthly EndNote class regularly had 2 or 3 attendees, the videos average twice that number in views per month. The Library hopes to develop more video series in the future, as it has become clear that the SIU-SOM community prefers and appreciates the convenience of on-demand viewing. Beyond videos, the Library is also reassessing its print collection and looking into electronic options for the most highly used reserve materials.

\section{CONCLUSION}

As was the case with most institutions, the COVID-19 pandemic disrupted the normal operating procedures of the SIU School of Medicine in significant ways. Quick and innovative strategies were required on all levels to respond to rapidly changing protocols and administrative directives. The library staff did a remarkable job of adapting service models and workflows to ensure that they could continue to provide quality information resources and assistance to the Medical School. While not every modification was perfect, several of the COVID-related changes actually improved library services and as such, will be made permanent. The Library will develop additional video tutorials, and continue to offer virtual classes and consultations. In the future, the focus on book purchases will be toward electronic books over print, however, pricing, licensing, and platform performance and features may impact those decisions.

This year has certainly presented the Medical Library with several unexpected and unprecedented challenges, but the pandemic also provided an opportunity for the Library to reassess its priorities, re-examine its strengths, and identify points for improvement. The Medical 
Library has demonstrated how important it is to the SIU-SOM community, and it is confident that it will emerge better than ever post-pandemic!

\section{REFERENCES}

1. Southern Illinois University Medical Library. COVID-19 (Coronavirus) [updated 5/06/2020; accessed 11/3/2019]. Available from: https://libguides.siumed.edu/c.php?g=1008569

2. Southern Illinois University Medical Library. Creating Educational Videos [updated 3/26/2020; accessed 11/3/2019]. Available from:

https://LibGuides.siumed.edu/videoproduction.

3. Brown R. PubMed Essentials 2020 (on-demand): Network of the National Library of Medicine; 2020 [8/5/2020; accessed 11/3/2020]. Available from: https://nnlm.gov/class/pubmed-essentials-2020-demand/23191.

4. Southern Illinois University Medical Library. Finding Full Text Articles [updated 8/3/2020; accessed 11/3/2020]. Available from: https://LibGuides.siumed.edu/fulltext.

5. Southern Illinois University Medical Library. EndNote [updated 8/3/2020; accessed 11/3/2020]. Available from: https://LibGuides.siumed.edu/endnote. 
Figure 1: Literature Search Requests Received by Month January 2018 - October 2020

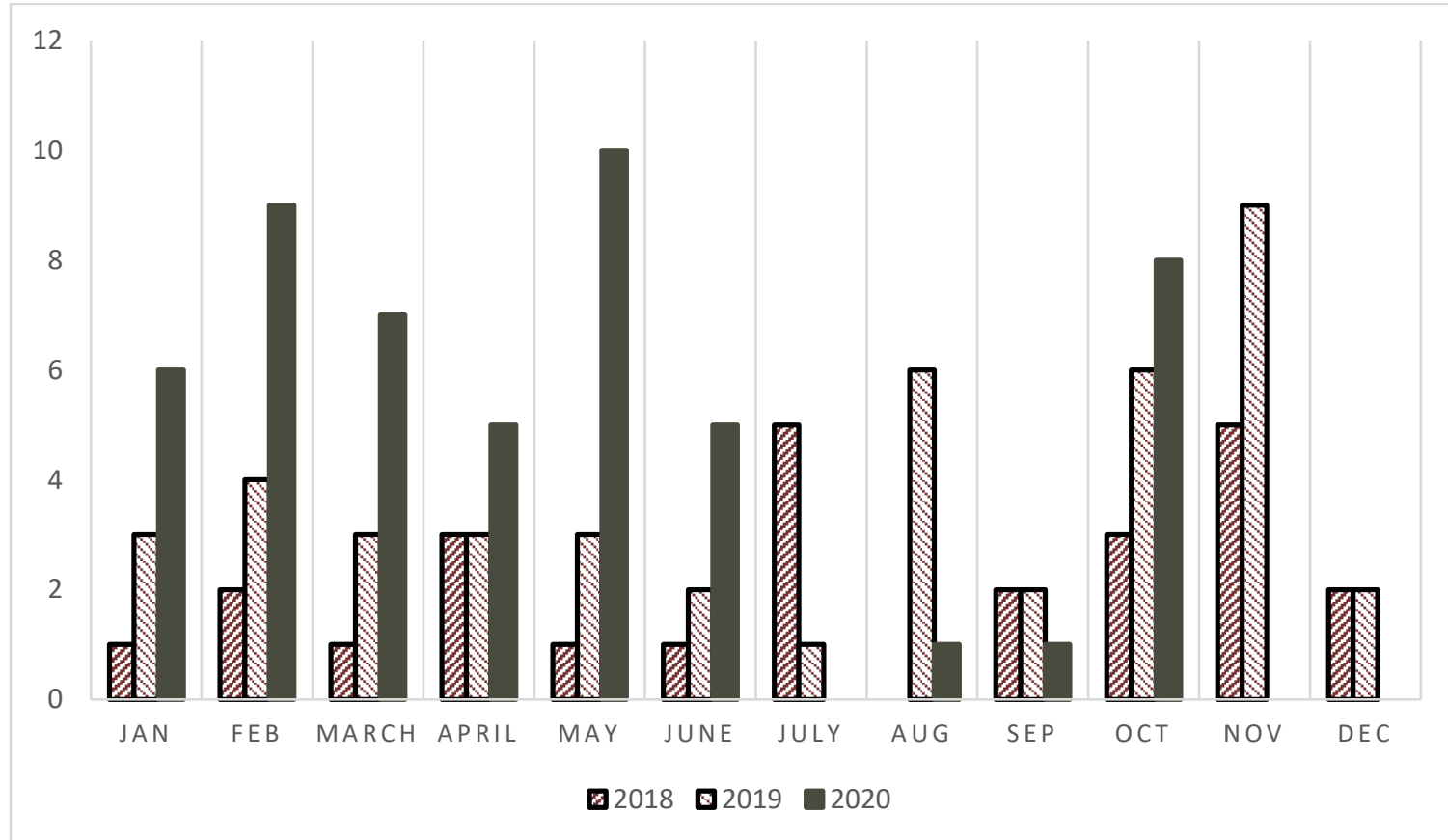


Table 1: Videos Developed During COVID-19

\begin{tabular}{|l|r|r|}
\hline \multicolumn{1}{|c|}{ Video } & Runtime & \multicolumn{2}{c|}{$\begin{array}{c}\text { Views } \\
\text { 7/31- } \\
\text { 10/31* }\end{array}$} \\
\hline Recording a PowerPoint as MP4 & $3: 33$ & 24 \\
\hline Full Text- Citation Linker & $5: 02$ & 21 \\
\hline Full Text- E-Journal List & $4: 05$ & 12 \\
\hline Full Text- Databases & $7: 03$ & 48 \\
\hline Full Text- SIU Carbondale & $6: 41$ & 13 \\
Resources & & 24 \\
\hline EndNote- Getting Started & $7: 03$ & 24 \\
\hline EndNote- Importing References & $9: 24$ & 24 \\
\hline EndNote- Organization/Resource & & 24 \\
Management & $7: 56$ & 24 \\
\hline EndNote- Syncing \& Sharing & $8: 36$ & 59 \\
\hline EndNote- Creating Citations & $6: 09$ & 26 \\
\hline Virtual Library Tour & $4: 10$ & 24 \\
\hline Library Website Tour & & \\
\hline
\end{tabular}

*Views as counted by Google Analytics integration with screencast.com 